\title{
The post-traumatic colour change of primary incisors: a colourimetric and longitudinal study
}

\author{
HONG-KEUN HYUN, TEO JEON SHIN \& YOUNG-JAE KIM
}

Department of Paediatric Dentistry, School of Dentistry, Dental Research Institute, Seoul National University, Seoul

National University Dental Hospital, Seoul, Korea

International Journal of Paediatric Dentistry 2016; 26: $291-300$

Background. Tooth colour change after trauma has been described subjectively as ranging from yellow/pink to grey/black.

Aim. To investigate the longitudinal colourimetric change of post-traumatic discoloured primary incisor using an intraoral colourimeter.

Design. A total of 34 primary incisors from 15 boys and eight girls were studied. The mean post-injury day during clinic visits (SD) and number of visit was 205.4 (194.8) and 3.9 (2.0). CIE $L^{*}$ (lightness), $a^{*}$ (green-red) and $b^{*}$ (blue-yellow) of the maxillary primary incisors were measured at every visit. The colour difference $\left(\Delta E^{*}{ }_{a b}\right)$ was calculated between the traumatized tooth and the control. Scatter graphs were made depicting the colour change of discoloured teeth and the $\Delta E_{a b}^{*}$ over time.

Results. Mean CIE $L^{*}, a^{*}$ and $b^{*}$ of the unaffected control were 80.8 (2.29), 0.9 (0.77) and 13.1 (2.67), respectively. $L^{*}$ gradually decreased to 70.7 (on day 71), then slowly recovered. $a^{*}$ increased to 3.7 (day 29) and decreased slowly. $b^{*}$ only demonstrated a small change that was within the control range during the follow-up. $\Delta E_{a b}^{*}$ increased to 9.58 (day 56) and decreased slowly.

Conclusion. The earlier recovery of $a^{*}$ was followed by the recovery of $L^{*}$. During the post-traumatic period, $\Delta E_{a b}^{*}$ failed to reach the clinically acceptable threshold.

\section{Introduction}

Traumatic injuries to the primary anterior teeth occur frequently ${ }^{1}$. Crown discolouration is one of the most common post-traumatic complications in children ${ }^{2-4}$. Intrapulpal haemorrhage often occurs as a result of traumatic injuries to the teeth ${ }^{3}$. Following haemorrhage, hemosiderin, which forms from haemoglobin degradation, penetrates into the dentinal tubules and can change the tooth's colour $^{5}$. Several prior studies have demonstrated that approximately $50 \%$ of discoloured (dark or grey) teeth can regain normal pigmentation if the vital pulp absorbs the blood pigments ${ }^{3}$.

If a tooth is persistently discoloured after trauma, radiographic and clinical signs may be unable to detect the development of periapical pathosis, which requires intervention ${ }^{3}$.

Correspondence to:

Young-Jae Kim, Department of Paediatric Dentistry, School of Dentistry, Seoul National University, 101, Daehakno,

Jongno-gu, 110-768 Seoul, Korea.

E-mail: neokarma@snu.ac.kr
Some have suggested that it is appropriate to perform extraction or pulp treatment for persistently discoloured teeth even before they develop clinical or radiographic signs of pathology ${ }^{6}$; however, more recently, others have recommended following patients (without intervention) unless necrosis or signs of infection are detected ${ }^{7,8}$. This approach is based upon the hypothesis that discoloured primary teeth may remain asymptomatic if the necrotic pulp is aseptic, or if it is infected by micro-organisms (of low virulence) that are suppressed by the host's immune system ${ }^{9}$.

Sometimes, discoloured teeth develop periapical pathosis that requires active intervention to prevent damage to the permanent teeth ${ }^{10}$. Inflammation surrounding primary dentition affected with apical pathosis can interfere with the germ tissue of the permanent teeth ${ }^{11}$. Therefore, it is important to initiate timely endodontic intervention when discoloured, traumatized primary teeth have signs of pulp necrosis including abscesses, fistulas, periapical lesions or inflammatory root resorption ${ }^{11}$. Tooth colour change is one of the most common reasons that parents seek 
treatment for their children after traumatic injuries, secondary to tooth displacement ${ }^{12}$. Therefore, it is essential that clinicians who see such patients have a reference for diagnosis in their clinical decision-making.

The previous studies that investigated tooth discolouration after trauma have described colours ranging from yellow/pink to grey/ black $^{13,14}$; however, the colour assessments in these studies were mostly subjective based on the naked eye or the use of a shade guide, rather than on colourimetric scales from calibrated reference instruments ${ }^{10,14}$. There are few studies that address the longitudinal colourimetric change of discoloured primary teeth after traumatic injury.

Recently, the CIE value was established using a scientific spot measurement type of intraoral colourimeter to determine the colour of the smooth surface of primary and permanent teeth ${ }^{15,16}$. The CIE (Commission Internationale de l'Eclairage) Lab colour system consists of three-dimensional colour coordinates. CIE $L^{*}$ represents lightness. The darkest black is indicated by 0 and the brightest white by 100 . CIE $a^{*}$ and $b^{*}$ indicate chromaticism. Positive values of CIE $a^{*}$ represent red, whereas negative values indicate green. Positive and negative values of $\operatorname{CIE~} b^{*}$ represent yellow and blue, respectively ${ }^{16}$. One study reported that the intraoral colourimeter had effective in vivo repeatability and could be used for more precise colourimetric measurement using colour quantification with CIELab coordinates $^{17}$. If the longitudinal colour course of traumatized teeth was established, one could develop diagnostic criteria to assess a tooth's current status and predict its prognosis, together with accompanying clinical and radiological examinations.

The aim of this study was to investigate the longitudinal colour changes of discoloured primary incisors caused by traumatic injury using an intraoral colourimeter.

\section{Materials and methods}

The study participants consisted of children with discolourations of their maxillary primary incisors caused by a traumatic injury who presented to the Department of Paediatric
Dentistry at the Seoul National University Dental Hospital in Seoul, Korea between January 2010 and January 2014. Exclusion criteria at the first visit included: a history of caries or restorations in the traumatized primary incisors, pulp exposure, pathologic/inflammatory root resorption other than atypical root resorption, severe tooth mobility $>1 \mathrm{~mm}$ in any direction, gingival swelling, gingival abscess or fistula, extensively symptomatic periapical lesion, need for emergent treatment (such as crown fracture or root fracture) and prior history of incisor discolouration. Children were also excluded if their caregiver did not agree to their participation. A total of 34 primary incisors from 15 boys and eight girls were included in this study. The study protocol was approved by the institutional review board of the School of Dentistry at Seoul National University (IRB No.: S-D20140044).

Data were collected from the participants' records including gender, type of injured primary incisor, type of injury, clinical/radiographic evaluation, age at the time of injury, and the time interval between the injury and the first observation of discolouration by parents or investigator. Every patient returned for periodic follow-up visits to provide timely and appropriate treatment whenever necessary. The interval between visits ranged from 1 to 6 months and was determined by the symptom severity. Clinical examinations were performed and recorded at every visit. Radiographic examinations were also performed for diagnostic purposes, if necessary, with permission from the participants' parents. Asymptomatic, traumatized teeth with partially/totally obliterated pulp or with mild external root resorption identified by radiographic examinations during the follow-up period were carefully observed without immediate treatment; however, if symptomatic, the tooth was treated endodontically or extracted depending on the severity of the signs. Some of the clinical signs and symptoms include swelling, sinus tract or suppuration of pus from the sulcus surrounding the affected tooth, and increased mobility/sensitivity to percussion, and radiographic evidence of inflammatory 
root resorption with a periapical radiolucent area.

The tooth colour was measured using the intraoral colourimeter ShadeEye NCC (Shofu, Tokyo, Japan) equipped with a disposable elastic tip. This elastic tip can measure $2.5 \mathrm{~mm}$ in diameter, with an outside diameter of $5.0 \mathrm{~mm}$ at the base and $4.0 \mathrm{~mm}$ at the top ${ }^{18}$. A pilot study performed by this group demonstrated that the intraclass correlation coefficient ranges of CIE $L^{*}, a^{*}$ and $b^{*}$ measured three times in twelve healthy incisors were 0.90-0.97, 0.89-0.97 and 0.92-0.98, respectively. These values reflect the device's high reproducibility. Our previous study, which investigated the maxillary primary incisor colour in healthy children, found that most labial colour differences among incisors from the same person were clinically acceptable ${ }^{15}$. The control colourimetric value was defined by the colours of adjacent unaffected incisors excluding the injured tooth out of four maxillary incisors. The colourimeter was calibrated before each measurement. The labial surface of the maxillary incisors was cleaned gently with a soft rubber cup before measurement. The examiner placed the spot measurement device on the middle third of the mesiodistal and cervicoincisal dimension of each tooth's labial surface. There was no gap between the elastic tip and the tooth's surface during the measurement. One experienced examiner performed all of the measurements using the same unit chair, in the same clinic room and with similar ambient light conditions.

Colour data were collected on the dimensions of the CIELab colour space. The colour difference $\left(\Delta E^{*}{ }_{a b}\right)$ between the traumatized tooth and the unaffected control teeth was calculated using the following equation:

$$
\Delta E_{a b}^{*}=\left[\left(\Delta L^{*}\right)^{2}+\left(\Delta a^{*}\right)^{2}+\left(\Delta b^{*}\right)^{2}\right]^{1 / 2}
$$

where

$$
\begin{aligned}
& \Delta L^{*}=L^{*}{ }_{T}-L_{C}^{*} ; \quad \Delta a^{*}=a^{*}{ }_{T}-a^{*}{ }_{C} ; \\
& \Delta b^{*}=b^{*}{ }_{T}-b^{*}{ }_{C}
\end{aligned}
$$

$L^{*}{ }_{T}, a_{T}^{*}, \quad b^{*}$ represent the colour measurements of the traumatized tooth, and $L^{*}{ }_{C}, a^{*}{ }_{C}$, $b{ }^{*}$ represent the average colour measure- ments for the adjacent control teeth from the same person. In case that all discolourations in four maxillary incisors were discovered, the colour difference was not calculated. The $\Delta E^{*}{ }_{a b}$ value of 3.3 (which has a $50 \%$ clinical acceptability threshold) was used to evaluate the significance of the colour difference as a reference value ${ }^{19}$.

Demographic data were analysed using SPSS 21 (IBM Corp., Somers, NY, USA). Scatter plots were made of the CIE values of the unaffected control teeth versus the child's age. Colourimetric values were compared to age using Pearson correlations. Scatter plots were made to represent the colour change of the traumatized teeth and the colour differences between the post-traumatic teeth and the adjacent control teeth. Trend lines showing the higher $R$ squared (goodness of fit) were chosen to interpret data in the scatter diagrams.

\section{Results}

Patient characteristics are presented in Table 1. The mean age at time of injury of subjects was 39.0 months (SD 12.8) and ranged between 15 and 71 months. The longest period of follow-up after injury was 941 days. The mean number of follow-up visits was 3.9 (2.0), which nine being the largest number. The average age at the final visit was 49.5 (16.7) months. The boy to girl ratio was $1.88: 1$.

With regard to discolouration among the maxillary primary teeth, the most affected tooth was the right primary central incisor $(47.1 \%)$, followed by the left primary central incisor $(35.5 \%)$, the right lateral incisors $(8.8 \%)$ and the left lateral incisors $(8.8 \%)$. The most common type of injury was subluxation $(76.5 \%)$. Intrusive luxation accounted for $23.5 \%$ of the injuries. The most frequent radiographic findings during follow-up were external root resorption including atypical root resorption $(20.6 \%)$, pulp canal obliteration $(11.8 \%)$ and periapical radiolucency $(11.8 \%)$. Endodontic treatment or extraction was performed in $14.7 \%$ of cases during the follow-up period.

Colour distributions of the control primary incisors are shown in Fig. 1. Mean CIE $L^{*}, a^{*}$ and $b^{*}$ of the unaffected control incisors were 


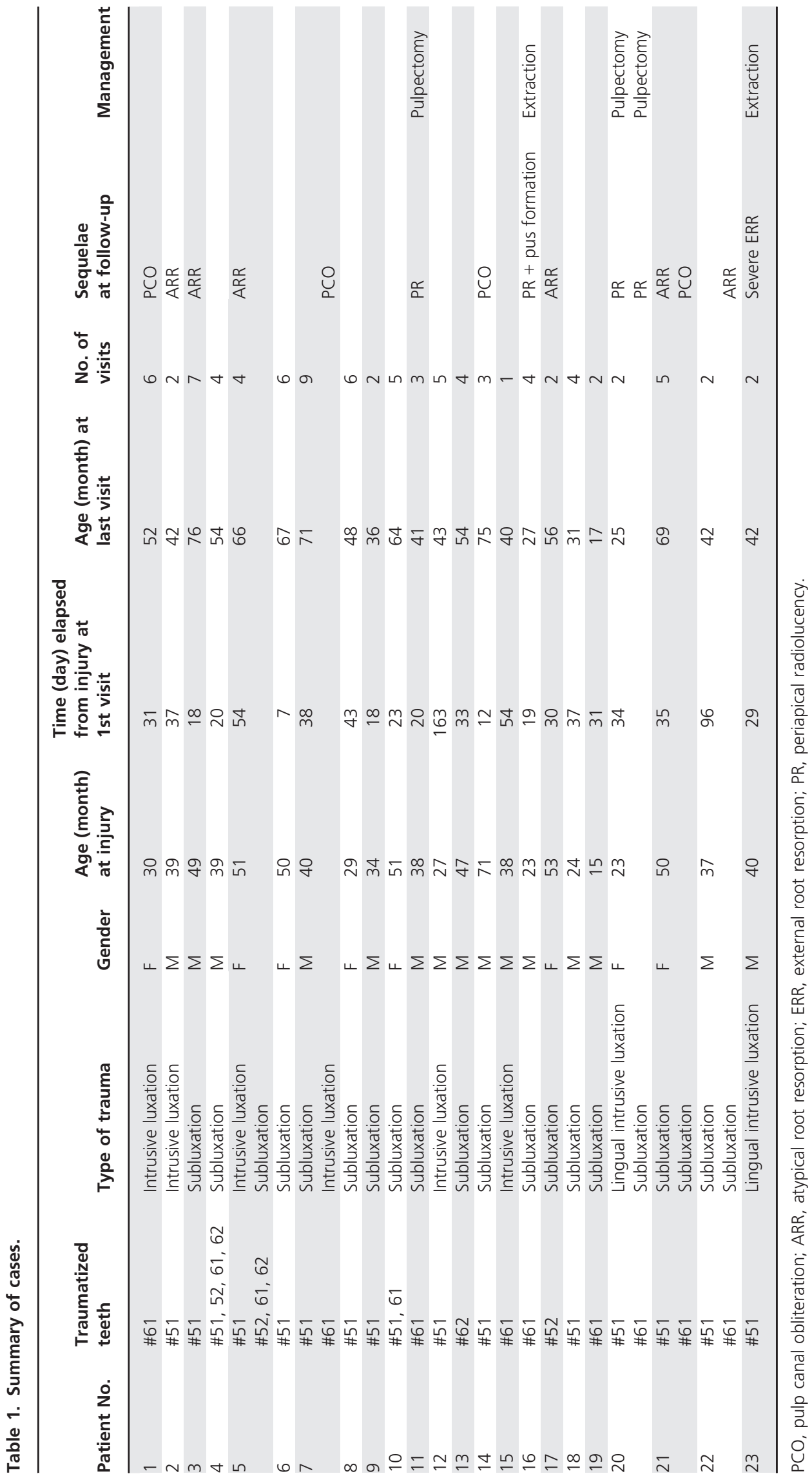




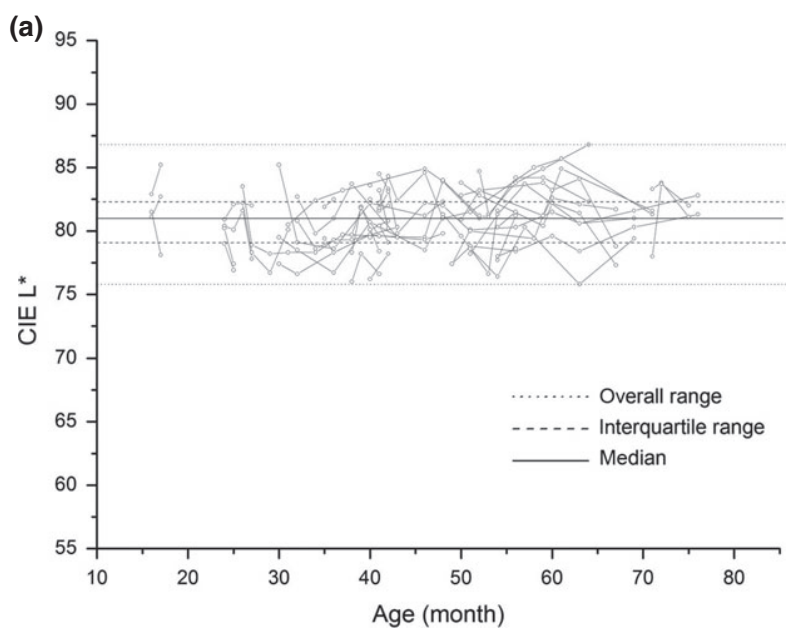

The time-dependent colourimetric changes of the traumatized incisors are shown in Fig. 2. A trend line for polynomials of a higher order was plotted on each graph. The trend line equations were calculated as follows:

$$
\begin{aligned}
\mathrm{CIE} L^{*}= & 3 \mathrm{E}-15 \mathrm{x}^{6}-1 \mathrm{E}-11 \mathrm{x}^{5} \\
& +1 \mathrm{E}-08 \mathrm{x}^{4}-5 \mathrm{E}-06 \mathrm{x}^{3} \\
& +0.0013 \mathrm{x}^{2}-0.1205 \mathrm{x} \\
& +74.216\left(R^{2}=0.1538\right) \\
\mathrm{CIE} a^{*}= & -9 \mathrm{E}-16 \mathrm{x}^{6}+3 \mathrm{E}-12 \mathrm{x}^{5} \\
& -3 \mathrm{E}-09 \mathrm{x}^{4}+1 \mathrm{E}-06 \mathrm{x}^{3} \\
& -0.0003 \mathrm{x}^{2}+0.0123 \mathrm{x} \\
& +3.5275\left(R^{2}=0.1702\right) \\
\mathrm{CIE} b^{*}= & 8 \mathrm{E}-16 \mathrm{x}^{6}-2 \mathrm{E}-12 \mathrm{x}^{5} \\
& +1 \mathrm{E}-09 \mathrm{x}^{4}-2 \mathrm{E}-07 \mathrm{x}^{3} \\
& -3 \mathrm{E}-05 \mathrm{x}^{2}+0.0128 \mathrm{x} \\
& +11.984\left(R^{2}=0.0967\right)
\end{aligned}
$$

where $\mathrm{x}$ is the number of days since the injury.

Within the limitations of the equation's low $R$-squared value, $L^{*}$ gradually decreased to 70.7 at 71 days and slowly recovered to 75.2 (on day 321). After having reached a plateau, the $L^{*}$ value began to enter the range of the control at 589 days. CIE $a^{*}$ increased to 3.7 on day 29 and then started to enter the control range at 95 days after decreasing slowly. In contrast, during the entire follow-up period, CIE $b^{*}$ had a relatively small change within the range of the control. It eventually increased to 16.6 at 652 days. In most individual cases, CIE $a^{*}$ changed and recovered earlier than did CIE $L^{*}$, consistent with the trend line.

Changes of the colour difference between the post-traumatic tooth and the control are shown in Fig. 3. The trend line equation was calculated using the following equation:

$$
\begin{aligned}
\Delta E_{a b}^{*}= & -2 \mathrm{E}-15 \mathrm{x}^{6}+5 \mathrm{E}-12 \mathrm{x}^{5} \\
& -6 \mathrm{E}-09 \mathrm{x}^{4}+3 \mathrm{E}-06 \mathrm{x}^{3} \\
& -0.0007 \mathrm{x}^{2}+0.0556 \mathrm{x} \\
& +8.2459\left(R^{2}=0.0796\right)
\end{aligned}
$$

tively. CIE $L^{*}$ in the control teeth was significantly correlated with age $(r=0.195$, $P=0.009)$, whereas CIE $a^{*}$ and $b^{*}$ were not $(P>0.05)$.

where $\mathrm{x}$ is the number of days elapsed from injury. 
Given the limitations of the low $R$-squared value of this equation, $\Delta E_{a b}^{*}$ increased to 9.58 on day 56 and then decreased slowly, never reaching the $50 \%$ clinical acceptability threshold $\left(\Delta E^{*} a b=3.3\right)$.

\section{Discussion}

Previous studies have shown that shortly following trauma, the crown develops a pink discolouration within 2-3 days $^{20}, 1-8$ days $^{21}$ or $1-3$ weeks ${ }^{4}$. Next, there is a shift from pink to grey/grey-blue which occurs within 6 days $^{21}$ or $2-3$ weeks ${ }^{22}$. Horan ${ }^{10}$ introduced the term 'dark discolouration' to represent various dark shades of crown discolouration after injury including grey, brown and black. Dark discolouration tends to persist for several weeks, and then, the crown recovers to its original shade or to a yellowish hue. In the present study, there was an increase in $a^{*}$ (which represents red when positive) earlier than there was a decrease in $L^{*}$ (which represents dark black when negative). Also, CIE $a^{*}$ returned to the control range in less time than $\operatorname{did} L^{*}$. CIE $b^{*}$, which represents a yellowish discolouration, stayed within the control range until 1 year after injury, when it increased slightly. Consequently, each sequential change in CIE colour coordinate could represent a linear progression of discolouration from pink to grey to yellow over time (Fig. 2).

The trend line of colour change demonstrated two peaks. One narrow peak occurred in the earlier period and another wide peak one occurred in the later time period of time. The overall difference decreased slowly during follow-up (Fig. 3). The early changes in CIE $L^{*}$ and $a^{*}$ may represent the initial colour differences after injury. In contrast, the change in CIE $b^{*}$ during the latter part of the period may represent a delayed colour change; however, the trend line of the overall colour difference failed to reach the clinical acceptability threshold $\left(\Delta E_{a b}^{*}=3.3\right)$.

In the present study, the mean elapsed day after injury (SD, range, lst range, median, 3rd quartile) was 205.4 days (194.8, 1-941, $54,149,282.8)$ in the post-traumatic period. Careful interpretation after approximately
300 days is still needed because this study did not have a large enough sample size. One study found that $38.7 \%$ of the children with traumatic dental injuries sought treatment on the day of the injury, whereas $37.8 \%$ did so 1-7 days after the injury ${ }^{23}$. Another study demonstrated that it took 2-3 weeks on average for patients to visit the dentist after a dental injury ${ }^{24}$. The interval between the injury and the presentation is likely to be shorter in cases in which there are easily recognizable traumas such as complete avulsion, displaced luxation and crown fracture; however, if the patient has a mild clinical presentation after trauma such as a concussion or subluxation with minimal displacement, then the presentation to dental care is likely to be postponed until crown discolouration appears. In our study, the average elapsed time between the injury and the first visit was 38.3 days (32.7), which corresponds to the colour difference of 9.5 according to the calculated trend line equation. We assume that most parents could perceive their child's tooth discolouration at the time of their first visit to the clinic.

Previous studies have reported that over $50 \%$ of traumatized primary teeth become discoloured $^{1,3}$. The most frequent types of injury for which patients seek treatment are concussion and loosening, followed by root fracture in case of the crown discolouration ${ }^{12}$. The most common type of injury in the present study was subluxation $(76.5 \%)$, followed by intrusive luxation $(23.5 \%)$. Cases of crown or root fracture requiring emergency treatment were excluded from the study.

Previous studies have demonstrated that there is little correlation between the degree of discolouration and the histopathologic changes in the pulp of the post-traumatic discoloured primary incisors ${ }^{14,25}$. Previous investigators reported that $55-80 \%$ of the discoloured primary incisors were retained with no treatment during the follow-up period or until their normal exfoliation time ${ }^{10,26,27}$; however, it has also been reported that some discoloured teeth develop periapical lesions including sinus tract and/or external inflammatory root resorption, indicating a necrotic and infected pulp ${ }^{10}$. In our 

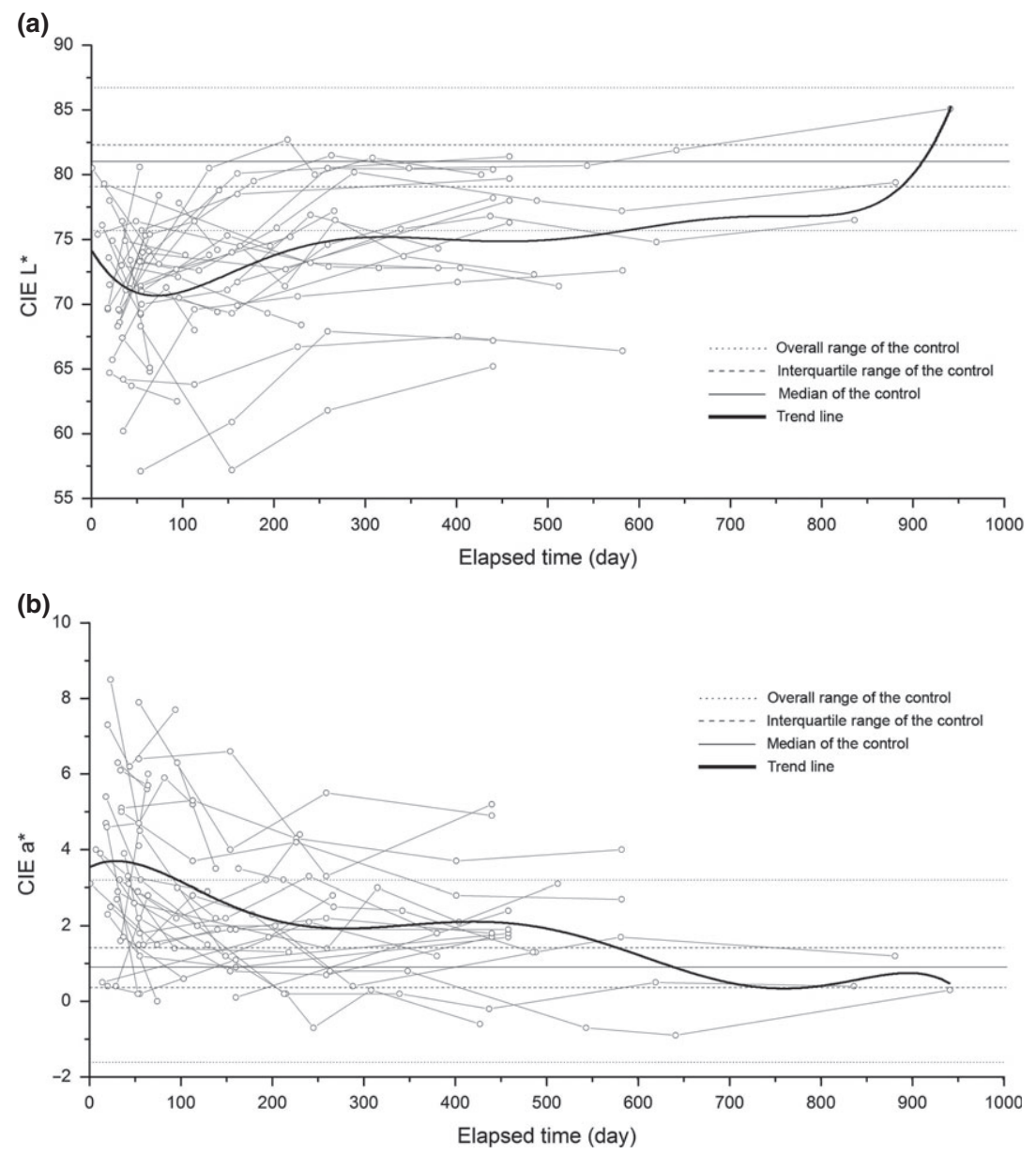

Fig. 2. Time courses of (a) CIE $L^{*}$ (b) $a^{*}$ and (c) $b^{*}$ values of the post-traumatic primary incisors. Horizontal line drawings show the colour range of the control.

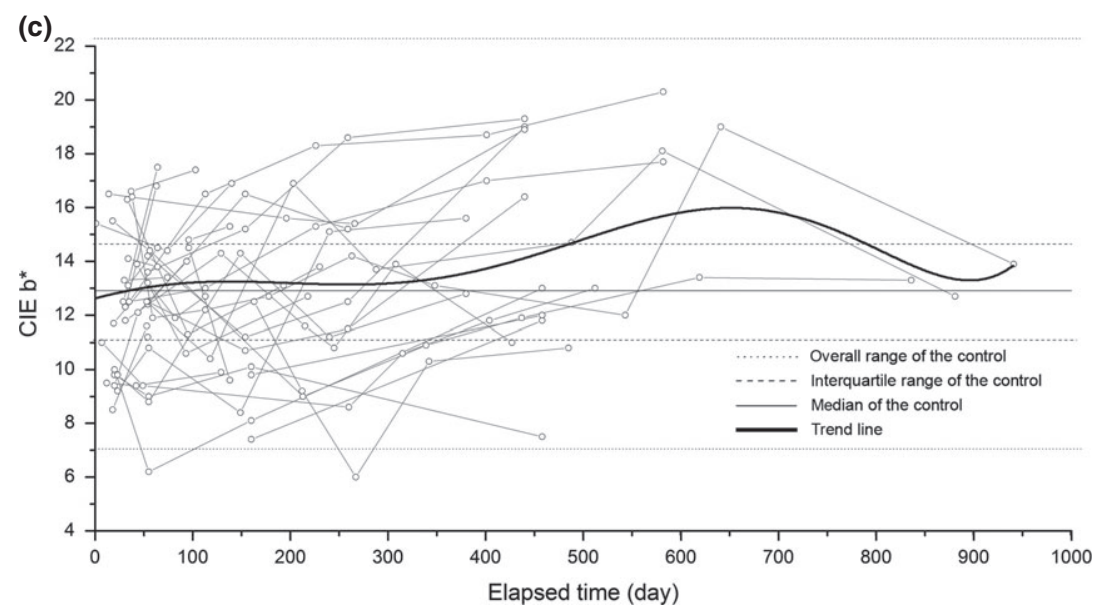

study, five of the 34 teeth (14.7\%) with post-traumatic crown discolouration developed periapical lesions requiring intervention.

Atypical root resorption (ARR) of the maxillary primary incisors is characterized by superficial external root resorption restricted to the sides of the apical half of the root length and the width of the coronal half ${ }^{10,28}$. The ARR mostly results from traumatic forces $^{28}$. External root resorption in permanent dentition is always considered a pathologic process; however, external resorption in primary teeth may be either physiologic, as part of normal dentition development, or pathologic, as a result of inflammation following traumatic injuries or pulpal infection ${ }^{10}$. Discoloured teeth are assumed to be aseptic if there are no clinical signs of infection in the 


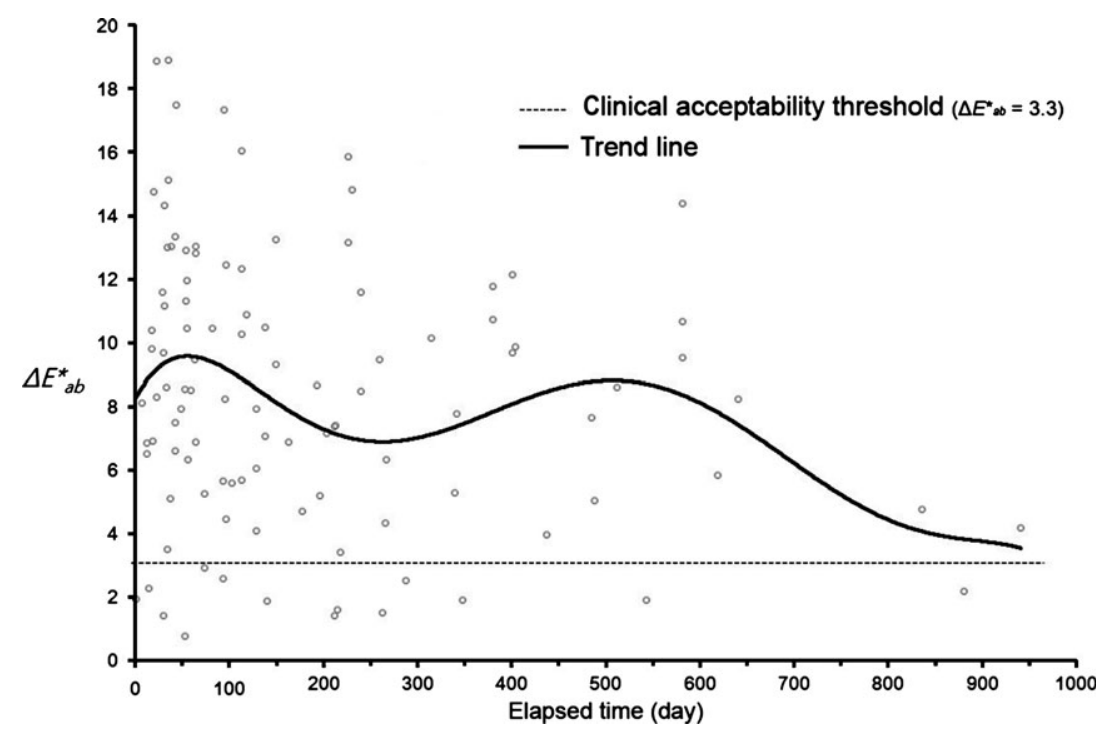

Fig. 3. Longitudinal changes in the colour difference between the traumatized and the control teeth. setting of radiographic signs such as surface external root resorption or expansion of the follicle of their permanent successors; however, if there is radiographic pathology that is accompanied by clinical signs such as swelling, sinus tracts, increased mobility or sensitivity to percussion, then the tooth is likely infected $^{29}$. Although asymptomatic primary incisors with crown discolouration may contain a necrotic or necrotizing pulp ${ }^{9}$, their permanent successors will not be harmed as long as the infections are contained within the pulp $^{26}$. Six of the 34 discoloured teeth $(17.6 \%)$ in this study were diagnosed with ARR and were carefully observed during the follow-up period.

Odontoblasts continuously form dentin throughout the life of a tooth; however, its production increases in the presence of a bacterial challenge or other stimuli including trauma ${ }^{3,30}$. Pulp canal obliteration (PCO) does not necessarily require endodontic treatment unless pulp necrosis has developed $^{3}$. In the present study, we collected CIE values of teeth $(n=4)$ that were radiographically diagnosed with PCO and those of control teeth at every visit $(n=14)$. The two groups were compared with an independentsample $t$-test $(\alpha=0.05)$. The CIE $L^{*}$ of the PCO group was significantly lower than was that of the control group $(P=0.017)$. In contrast, there were no statistical differences between the CIE $a^{*}$ and $b^{*}$ between the two groups $(P>0.05)$. Therefore, the PCO group was significant different from the control group with regard to the change in the lightness value. An average colour difference of 3.76 was calculated, which indicates that the colour change is perceivable by the naked eye $\left(\Delta E_{a b}^{*}>3.3\right)$. The literature suggests that teeth with pulp canal obliteration have decreased translucency combined with a yellowish or dark discolouration ${ }^{30}$. In the present study, however, there was little significant difference between the CIE $b^{*}$ (which represents yellow/blue colour) of the PCO teeth and control teeth. There was a significant decrease in the CIE $L^{*}$ of the PCO teeth, which may reflect decreased translucency.

According to a previous study ${ }^{10}$, although $43 \%$ of the traumatized primary incisors presented with signs of a necrotic and infected pulp, only $8 \%$ of these teeth developed swelling or sinus tracts indicative of infection/ necrosis. This finding suggests that early endodontic treatment is not necessary for discoloured teeth, but that close follow-up for the development of infection and root resorption is sufficient. In this study, the discoloured tooth was evaluated clinically and radiographically at every visit. Careful observation, rather than invasive management, was used when diagnosed with ARR or PCO with no pathologic signs; however, if a tooth was diagnosed with a periapical lesion and 
was a possible candidate for pulp therapy, then pulp canal treatment using a calcium hydroxide and iodoform paste was performed. This intervention was justified considering the tooth's remaining life expectancy and the root status of the symmetrical tooth in the opposite quadrant. A tooth was extracted if the pathological process accelerating its root resorption (based on clinical or radiographic assessment) because this could negatively affect the permanent tooth bud.

The incidence of subluxation in primary dentition in the setting of trauma has been reported over a broad range between $12 \%{ }^{31}$ and $40 \%^{32}$. The most frequent type of trauma in this study was subluxation (26/34), including two teeth with PCO, four with ARR, 17 with no signs, and three with periapical lesions requiring treatment. Our results are consistent with those of a previous study that found that $80.2 \%$ of 207 traumatized primary incisors with subluxation required no treatment, whereas $9.2 \%$ of them were extracted ${ }^{13}$.

There were six teeth in this study with intrusive luxation (6/34). Two had PCO, two had ARR, and two had no signs, requiring no intervention during the follow-up period; however, the two teeth with lingual intrusion $(2 / 34)$ were both treated either because of a periapical lesion or severe external root resorption. The primary incisor is frequently forced through the labial bone, away from the succedaneous tooth germ, because its root apex has a labial curvature ${ }^{33}$. A retrospective study of 172 intruded primary incisors reported that more than $80 \%$ of the teeth were pushed labially and most intruded teeth re-erupted ${ }^{34}$. They also found that $68 \%$ of the intruded incisors survived more than 3 years after the injury ${ }^{34}$. Nevertheless, the lingually directed traumatic impact forces the tooth root palatally, possibly resulting in contact with the permanent tooth follicle, which may negatively affect the root of the intruded tooth and the developing tooth germ $^{35}$. Therefore, discoloured teeth with lingual intrusion might adversely affect their apical root areas.

The intraoral colourimeter used in this study was reported to represent CIE values with high accuracy and reproducibility. It also has a suitable tip size to measure the value on the labial surface of the primary incisor ${ }^{15,16}$; however, the CIE Lab results from the present study may not be considered as the absolute reference values because there may be variability depending on the different type of measuring devices. Nevertheless, the results presented in this study may be helpful to understand the characteristics of the colour change of primary incisors after trauma. In addition, the colourimetric values might be a useful reference to evaluate the recovery of the discolouration over time (if using the same device used here). Regardless of a favourable recovery with regard to the colourimetric value, pathologic signs discovered during follow-up require immediate intervention to prevent damage to the succedaneous tooth germ.

This study had a low $R$-squared value calculated from the equation of the trend line for colourimetric value. One prior study identified that the $R$-squared value was a weak measure of importance in studies with small sample sizes ${ }^{36}$. Nonetheless, our results are not generalizable. Further studies are required to determine a reliable reference value and to interpret the changes in the colourimetric value based on large sample sizes. Still, the data presented here may suggest the overall pattern of tooth colour change over time after injury.

\footnotetext{
Why this paper is important to paediatric dentists

- Post-traumatic colour change of primary incisor crown is one of the common reasons for visits to the dental clinic.

- Paediatric dentists should understand the longitudinal colourimetric changes of post-traumatic discoloured teeth without developing periapical pathosis.

- Using an intraoral colourimeter could be helpful to develop diagnostic criteria to assess the discoloured tooth's current status and predict its prognosis, together with accompanying clinical and radiological examinations.
}

\section{Acknowledgements}

This study was supported from the overseas training program of Seoul National University Dental Hospital. 


\section{Conflict of interest}

The authors have no commercial interests to disclose.

\section{References}

1 Andreasen JO, Ravn JJ. Epidemiology of traumatic dental injuries to primary and permanent teeth in a Danish population sample. Int J Oral Surg 1972; 1: 235-239.

2 Andreasen JO. Luxation of permanent teeth due to trauma. A clinical and radiographic follow-up study of 189 injured teeth. Scand J Dent Res 1970; 78: 273286.

3 Borum MK, Andreasen JO. Sequelae of trauma to primary maxillary incisors. I. Complications in the primary dentition. Endod Dent Traumatol 1998; 14: 31-44.

4 Aguiló L, Gandía JL. Transient red discolouration: report of case. ASDC J Dent Child 1998; 65: 346348.

5 Arens D. The role of bleaching in esthetics. Dent Clin North Am 1989; 33: 319-336.

6 Johnson R. Traumatic dental injuries in children. Part I. Evaluation of traumatic dental injuries and treatment of injuries to primary teeth. Update Pediatr Dent 1989; 2: 1-4.

7 Harding AM, Camp JH. Traumatic injuries in the preschool child. Dent Clin North Am 1995; 39: 817-835.

8 Levine N. Injury to the primary dentition. Dent Clin North Am 1982; 26: 461-480.

9 Holan G, Fuks AB. The diagnostic value of coronal dark-gray discolouration in primary teeth following traumatic injuries. Pediatr Dent 1996; 18: 224-227.

10 Holan G. Development of clinical and radiographic signs associated with dark discoloured primary incisors following traumatic injuries: a prospective controlled study. Dent Traumatol 2004; 20: 276287.

11 Cardoso M, de Carvalho Rocha MJ. Association of crown discolouration and pulp status in traumatized primary teeth. Dent Traumatol 2010; 26: 413-416.

12 Garcia-Godoy F, Garcia-Godoy F, Garcia-Godoy FM. Reasons for seeking treatment after traumatic dental injuries. Endod Dent Traumatol 1989; 5: 180-181.

13 Fried I, Erickson P, Schwartz S, Keenan K. Subluxation injuries of maxillary primary anterior teeth: epidemiology and prognosis of 207 traumatized teeth. Pediatr Dent 1996; 18: 145-151.

14 Soxman JA, Nazif MM, Bouquot J. Pulpal pathology in relation to discolouration of primary anterior teeth. ASDC J Dent Child 1984; 51: 282-284.

15 Hyun HK, Lee YK, Kim YJ et al. Colour distribution of maxillary primary incisors in Korean children. Color Res Appl 2010; 35: 153-158.

16 Bak SY, Kim YJ, Hyun HK. Colour change of white spot lesions after resin infiltration. Color Res Appl 2014; 39: 506-510.
17 Karaagaclioglu L, Terzioglu H, Yilmaz B, Yurdukoru B. In vivo and in vitro assessment of an intraoral dental colourimeter. J Prosthodont 2010; 19: 279-285.

18 Tung FF, Goldstein GR, Jang S, Hittelman E. The repeatability of an intraoral dental colourimeter. J Prosthet Dent 2002; 88: 585-590.

19 Um CM, Ruyter IE. Staining of resin-based veneering materials with coffee and tea. Quintessence Int 1991; 22: 377-386.

20 Andreasen FM. Pulpal healing after luxation injuries and root fracture in the permanent dentition. Endod Dent Traumatol 1989; 5: 111-131.

21 Jacobsen I. Criteria for diagnosis of pulp necrosis in traumatized permanent incisors. Scand J Dent Res 1980; 88: 306-312.

22 Malmgren B, Hübel S. Transient discolouration of the coronal fragment in intra-alveolar root fractures. Dent Traumatol 2012; 28: 200-204.

23 Garcia-Godoy F, Garcia-Godoy F, Garcia-Godoy FM. Primary teeth traumatic injuries at a private pediatric dental center. Endod Dent Traumatol 1987; 3: 126-129.

24 Kenwood M, Seow WK. Sequelae of trauma to the primary dentition. J Pedod 1989; 13: 230-238.

25 Croll TP, Pascon EA, Langeland K. Traumatically injured primary incisors: a clinical and histological study. ASDC J Dent Child 1987; 54: 401-422.

26 Sonis AL. Longitudinal study of discoloured primary teeth and effect on succedaneous teeth. J Pedod 1987; 11: 247-252.

27 Reed AJ 3rd, Sayegh FS. The dark primary incisor. Dent Surv 1978; 54: 16-19.

28 Holan G, Yodko E, Sheinvald-Shusterman K. The association between traumatic dental injuries and atypical external root resorption in maxillary primary incisors. Dent Traumatol 2015; 31: 35-41.

29 Holan G. Long-term effect of different treatment modalities for traumatized primary incisors presenting dark coronal discolouration with no other signs of injury. Dent Traumatol 2006; 22: 14-17.

30 Abbott PV. Aesthetic considerations in endodontics: internal bleaching. Pract Periodontics Aesthet Dent 1997; 9: 833-840.

31 Ferguson FS, Ripa LW. Prevalence and type of traumatic injuries to the anterior teeth of preschool children. J Pedod 1979; 4: 3-8.

32 Meadow D, Lindner G, Needleman H. Oral trauma in children. Pediatr Dent 1984; 6: 248-251.

33 Ravn JJ. Developmental disturbances in permanent teeth after intrusion of their primary predecessors. Scand J Dent Res 1976; 84: 137-141.

34 Holan G, Ram D. Sequelae and prognosis of intruded primary incisors: a retrospective study. Pediatr Dent 1998; 21: 242-247.

35 Gupta M. Intrusive luxation in primary teeth review of literature and report of a case. Saudi Dent J 2011; 23: 167-176.

36 McKay MD, Fitzgerald MA, Beckman RJ. Sample size effects when using $\mathrm{R}^{2}$ to measure model input importance. LANL technical report LA-UR-99-1357, Los Alamos National Laboratory, Los Alamos, NM, USA. 